\title{
Regularization Strategies for Discontinuity-Preserving Optical Flow Methods
}

\author{
Nelson Monzón, Agustín Salgado, and Javier Sánchez,
}

\begin{abstract}
The aim of this work is to study several strategies for the preservation of flow discontinuities in variational optical flow methods. We analyze the combination of robust functionals and diffusion tensors in the smoothness assumption. Our study includes the use of tensors based on decreasing functions, which has shown to provide good results. However, it presents several limitations and usually does not perform better than other basic approaches. It typically introduces instabilities in the computed motion fields in the form of independent blobs of vectors with large magnitude.

We propose two alternatives to overcome these drawbacks: first, a simple approach that combines the decreasing function with a minimum isotropic smoothing; second, a method that looks for the best parameter configuration that preserves the important motion contours and avoid instabilities. It relies on the input images and the regularization parameter. It is fully automatic, providing a near-optimal value for many sequences, as shown in the experiments. Both proposals allow to detect the contours of the motion field and produce more stable solutions for a large range of parameters. In the experimental results, we present a detailed study and comparison of the different strategies.
\end{abstract}

Index Terms-Optical Flow, Discontinuity-preserving, Matrixvalue Diffusion.

\section{INTRODUCTION}

$\mathbf{T}$ HE preservation of motion discontinuities is a traditional problem in variational optical flow methods, for which a reliable solution does not exist yet.

After the seminal work of Horn and Schunck [1], many works have emerged to solve this problem. Earlier solutions rely on directional information [2], [3] to steer the regularization according to image structures. In this line, and more recently, some methods, [4]-[6], combine diffusion tensors with robust functionals in order to avoid oversegmenting the flow field. It is also typical the use of anisotropic diffusion, [7]-[11], to reduce the effect of outliers and produce sharp motion edges.

Another alternative is to mitigate the diffusion at image contours by means of exponential functions [12]. This strategy is simple to implement, and it is currently used in many works in combination with robust functionals [13]. Other smoothing strategies include bilateral filtering [14] or nonlocal regularizations, like in [15] and [16].

All of these strategies present different difficulties to deal with motion contours. In many cases, it is challenging to chose the correct parameters that provide the best results.

The authors are with the Department of Computer Science, University of Las Palmas de Gran Canaria, Las Palmas de Gran Canaria, 35017 Spain email: nmonzon@ctim.es, $\{$ asalgado, jsanchez $\} @$ dis.ulpgc.es.
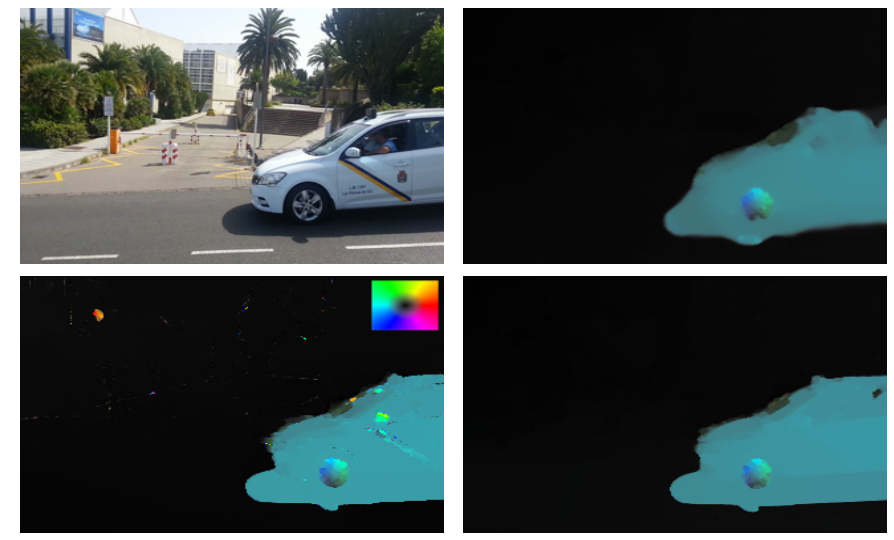

Fig. 1. Instability problem. Top row: sequence of a moving taxi and the solution obtained with the Brox et al. [10] method. Bottom row: instabilities appearing in discontinuity-preserving methods due to a wrong parameter setting (with the color scheme used to represent the motion fields in the upper-right corner) and an example of well-preserved discontinuities in the optical flow.

In this work, we analyze the behavior of several regularization strategies. In particular, we are interested in the combination of robust functionals and diffusion tensors, including the use of decreasing functions. The main benefits of these approaches are well known: they create piecewise-continuous motion fields, dealing with outliers and textured areas.

One of the problems of using decreasing functions, in the smoothness process, is that they easily introduce instabilities in the computed flow fields [17]. Figure 1 depicts an example of this situation. We observe blobs around the object contours in the bottom left image. The last image shows a good example when the parameter is correctly chosen.

However, most of the methods assign an empirical value to the parameters. Unfortunately, the configuration that better preserves discontinuities are prone to produce instabilities. These appear because the smoothness process is canceled at high image gradients.

We propose two mechanisms for avoiding these instabilities, which also facilitate the parameter choice. Our first approach ensures a minimum isotropic regularization. This is simply achieved by using a small constant in addition to the decreasing function. A similar idea was introduced in [18] but, in that case, the regularization was carried out for each component of the optical flow independently. This strategy allows to respect the motion discontinuities and is much more stable than the basic scheme.

The second approach automatically computes a parameter value that avoids stopping the diffusion. It is calculated from 
the gradient of the image and the regularization parameter. The benefit is that it is automatically adapted to the image range, at the same time that it preserves the motion contours with high accuracy.

We also analyze a scheme similar to the robust diffusion tensors proposed in [5] or [6]. The diffusion across image contours is controlled with a robust decreasing function and maintained along the isocontours of the objects. In this case, the method is very stable with respect to the anisotropic parameter.

This work follows our previous conference articles [17], [19]. The new contributions are, on the one hand, the comparison of all the strategies together, including the new method based on the robust diffusion tensor. This covers a representative set of discontinuity-preserving diffusion classes. On the other hand, we have also improved our proposal in [19], with a more reliable way of estimating the decreasing parameter. This allows detecting motion discontinuities in a more robust way. Other contributions are the extensive validation and justification of these approaches in the theoretical framework and experiments. We have also extended our method to multichannel images.

In Sec. III, we introduce the energy model and the different regularization strategies used in our study. Our energy functional derives from the Brox et al. [10] method and introduces a diffusion tensor. We explain each proposal in detail. The experimental results, in Sec. IV, deepen in the study of the different methods and the new proposals. Finally, a summary of the main ideas and conclusions in Sec. V.

\section{RELATED WORK}

Many ideas for preserving the optical flow contours come from the field of image denoising and regularization. For instance, Perona and Malik [20] proposed an anisotropic model for regularizing an image while preserving its discontinuities. On the other hand, Rudin, Osher and Sethian [21] proposed to minimize the Total Variation (TV) of an image with an attachment to the original image. This leads to a diffusion equation that reduces the image noise, yielding sharp edges. Some improvements on the Perona-Malik model are given in Black et al. [22]. This establishes the relation between this type of anisotropic diffusion processes, robust statistics and the minimization of energy functionals.

A review on different strategies for diffusion filtering in image regularization and restoration is in [23], [24]. The author introduces the theory underlying the use of diffusion tensors in image filtering, e.g., the structure tensor [25]. Another source of inspiration, for discontinuity-preserving in optical flow, is related with bilateral filtering, introduced by Tomasi and Manduchi [26]. In this case, the idea is to regularize an image using the information of the pixels that are near the actual position and have similar intensities or colors.

In the field of motion estimation, one of the former approaches to deal with discontinuities is due to Nagel and Enkelmann [2]. In this case, the regularization process is steered by a diffusion tensor that depends on the image gradient. Proesmans et al. [7] introduced the Perona-Malik
[20] anisotropic scheme in the estimation of optical flow. In a similar way, Black and Anandan [8], [27] used this type of anisotropic regularization, establishing the relation with robust statistics. They also extended the use of these type of functionals to the whole energy terms, turning the method more robust against outliers. They showed that this strategy deals with image noise at the same time that it preserves flow edges. On another hand, a TV scheme, like in [21], was introduced in optical flow by Cohen [9].

The method by Alvarez et al. [12] introduced a decreasing function to inhibit the smoothing at image contours. More recently, several methods use this kind of mitigating strategy in combination with robustification functionals, such as in [28], [29]. These type of smoothing strategies are the main focus of our study.

The use of continuous $L^{1}$ functionals was generalized in [10]. On the other hand, non-continuous $L^{1}$ functionals have also been used in Zach et al. [11] relying on a dual formulation, which yields a very efficient numerical scheme. Although these approaches are similar, they provide very different results, as can be seen in the online works [30] and [31], respectively.

Some examples in the use of diffusion tensors with robustification functions are given in [6], [32]. In this case, the authors introduce a motion tensor in the data term and a regularization tensor in the smoothness term, which are designed in a similar way. These take into account not only the variation of image intensities but also the variation of image gradients. It uses a quadratic penalizer for the diffusion along the contours, while a Perona-Malik diffusivity is used for mitigating the diffusion across flow edges. A similar strategy has also been used in [5], using the robust Huber norm.

The idea of bilateral filtering has been introduced in optical flow by Yoon and Kweon [33] and Xiao et al. [14]. In this case, the authors propose to regularize the flow field depending on the proximity and similarity of the intensities and flow values. In fact, this has to be seen as an extended trilinear filtering. Bilateral filtering has been used more recently, in combination with a TV- $L^{1}$ approach, in [15]. Bilateral filtering is very similar to using decreasing functions based on the image gradient. The main difference is the use of non-local information.

Additionally, the use of weighted median filters [34], as a post-processing step, allows to preserve fine motion details. However, it is very slow and is somehow redundant with respect to the regularization process.

There exist other strategies for improving the flow discontinuities. For instance, some authors propose to compute the optical flow at the same time that the objects or the motion are segmented [35]-[37]. The segmentation provides more information about the edges.

\section{THEORETICAL FRAMEWORK}

Given two multi-channel images, $I_{1}^{c}, I_{2}^{c}: \Omega \subset \mathbb{R}^{2} \rightarrow \mathbb{R}^{c}$, $\left\{I^{c}\right\}_{c=1, \ldots, C}$ and $C$ the number of channels, the optical flow, $\mathbf{w}=(u(\mathbf{x}), v(\mathbf{x}))^{T}$, puts in correspondence the pixels of the first image with the pixels of the second, with $\mathbf{x}=(x, y)^{T} \in$ 
$\Omega$. Functions $u(\mathbf{x})$ and $v(\mathbf{x})$ are the horizontal and vertical displacements, respectively.

Variational optical flow techniques rely on a global energy functional. Standard approaches typically include an attachment, $\mathcal{A}$, and a regularization term, $\mathcal{R}$, as follows:

$$
\begin{aligned}
E(\mathbf{w}) & =\int_{\Omega} \mathcal{A}\left(I_{1}, I_{2}, \nabla I_{1}, \nabla I_{2}, \mathbf{w}\right) \mathbf{d} \mathbf{x} \\
& +\alpha \int_{\Omega} \mathcal{R}\left(\nabla I_{1}, \nabla u, \nabla v\right) \mathbf{d} \mathbf{x} .
\end{aligned}
$$

In our case, the attachment term reads as

$$
\begin{array}{r}
\mathcal{A}\left(I_{1}, I_{2}, \nabla I_{1}, \nabla I_{2}, \mathbf{w}\right):=\Psi\left(\sum_{c=1}^{C}\left(I_{2}^{c}(\mathbf{x}+\mathbf{w})-I_{1}^{c}(\mathbf{x})\right)^{2}\right) \\
+\gamma \Psi\left(\sum_{c=1}^{C}\left|\nabla I_{2}^{c}(\mathbf{x}+\mathbf{w})-\nabla I_{1}^{c}(\mathbf{x})\right|^{2}\right),
\end{array}
$$

with $\Psi\left(s^{2}\right)=\sqrt{s^{2}+\epsilon^{2}}$ and $\epsilon:=0.001$ a small constant.

The regularization term is responsible for the continuity of the computed optical flow. In the next section we study several strategies.

\section{A. Regularization strategies}

We compare six diffusion schemes: Brox et al. [10], NagelEnkelmann [2], a robust anisotropic diffusion tensor (in the same way as [5]) and three variants of $\mathcal{R}\left(\nabla I_{1}, \nabla u, \nabla v\right):=$ $\Psi\left(g\left(\left|\nabla I_{1}\right|\right) \cdot\left(|\nabla u|^{2}+|\nabla v|^{2}\right)\right)$.

Table I shows some well known regularisers in the literature, their formulation and their main features. In Zimmer et al. [6], the regulariser is decomposed in eigenvalues $\left(\mu_{1}, \mu_{2}\right)$ and eigenvectors $\left(r_{1}, r_{2}\right)$ of the following structure tensor: $\mathbf{R}_{\rho}:=\sum_{c=1}^{3} K_{\rho} *$ $\left[\theta_{0}^{c}\left(\nabla_{2} I^{c} \nabla_{2}^{T} I^{c}\right)+\gamma\left(\theta_{x}^{c}\left(\nabla_{2} I_{x}^{c} \nabla_{2}^{T} I_{x}^{c}\right)+\theta_{y}^{c}\left(\nabla_{2} I_{y}^{c} \nabla_{2}^{T} I_{y}^{c}\right)\right)\right]$.

1) Brox approach:

$$
\mathcal{R}(\nabla u, \nabla v):=\Psi\left(|\nabla u|^{2}+|\nabla v|^{2}\right) .
$$

The Brox method proposes a robustification variant of the Horn-Schunck regulariser. The Total Variation function creates piecewise and continuous motion regions, but, at the same time, it creates rounded and dislocated contours.

In Fig. 2 we can see the effect of robustification and how the diffusion process is cut off with respect to the Horn-Schunck flow field.

\section{2) Regularization with decreasing scalar functions $(D F)$ :}

$$
\mathcal{R}\left(\nabla I_{1}, \nabla u, \nabla v\right):=\Psi\left(e^{-\lambda\left|\nabla I_{1}\right|} \cdot\left(|\nabla u|^{2}+|\nabla v|^{2}\right)\right) .
$$

This approach combines Total Variation and a decreasing function for mitigating the regularization at object contours.

We can increase the smoothing strength, in order to obtain more continuous flows, at the same time that it respects motion edges. Nevertheless, this decreasing function may cancel the smoothing term, creating instabilities in the flow field [17].
3) Regularization with decreasing scalar functions and constant diffusion $(D F-\beta)$ :

$\mathcal{R}\left(\nabla I_{1}, \nabla u, \nabla v\right):=\Psi\left(\left(e^{-\lambda\left|\nabla I_{1}\right|}+\beta\right) \cdot\left(|\nabla u|^{2}+|\nabla v|^{2}\right)\right)$.

One way to overcome the instabilities that may generate the $D F$ strategy, is to introduce a constant $\beta$, to ensure a minimum diffusion. As a consequence, the smoothness term is not completely canceled. A deeper study of this approach is realized in [17], where an empirical value for this constant was established as $\beta=0.001$. We use this setting in our tests.

4) Regularization with automatic setup of decreasing scalar functions (DF-Auto):

$$
\mathcal{R}\left(\nabla I_{1}, \nabla u, \nabla v\right):=\Psi\left(e^{-\lambda_{\pi}\left|\nabla I_{1}\right|} \cdot\left(|\nabla u|^{2}+|\nabla v|^{2}\right)\right) .
$$

This is the second mechanism to improve the $D F$ stability. This scheme differs from $D F$ in that the value of the parameter $\lambda_{\text {auto }}$ is automatically computed. The idea is to adapt its value when we detect a possible instability.

The minimization of (1), with the regularisers proposed in this work, provides a solution in the form

$$
\begin{aligned}
& 0=\partial_{u} \mathcal{A}-\alpha \operatorname{div}\left(\Psi^{\prime} \cdot \nabla u\right), \\
& 0=\partial_{v} \mathcal{A}-\alpha \operatorname{div}\left(\Psi^{\prime} \cdot \nabla v\right) .
\end{aligned}
$$

The instability normally arises when $\alpha \cdot \Psi^{\prime} \cong 0$. The amount of diffusivity is, thus, given by

$$
\alpha \cdot \Psi^{\prime}=\frac{\alpha \cdot e^{-\lambda\left|\nabla I_{1}\right|}}{\sqrt{e^{-\lambda\left|\nabla I_{1}\right|} \cdot\left(|\nabla u|^{2}+|\nabla v|^{2}\right)+\epsilon^{2}}} .
$$

This situation can be easily detected by checking if the numerator is close to zero. Then, one way to avoid instabilities is to ensure the following condition:

$$
\alpha e^{-\lambda\left|\nabla I_{1}\right|} \geq \xi>0
$$

with $\xi$ a small constant to avoid the cancelation of the divergence term. In our experiments, we fix its value to $\xi:=0.05$. Therefore, we may calculate $\lambda$ at the beginning of the process, to ensure this condition. Note that the gradient of the image is a function of $\mathbf{x}$. Then, from (3) we deduce a value of $\lambda$ for each position as

$$
\lambda(\mathbf{x}):=\frac{-\ln (\xi)+\ln (\alpha)}{\left|\nabla I_{1}(\mathbf{x})\right|}
$$

with $\left|\nabla I_{1}(\mathbf{x})\right|=\max _{c}\left\{\left|\nabla I_{1}^{c}(\mathbf{x})\right|\right\}$.

When the magnitude of the gradient is close to zero, $\lambda$ tends to $\infty$. In this way, the pixels located in homogeneous regions are considered as image edges. Thus, it is necessary to define a threshold that discriminates when a pixel belongs to an edge or not.

We propose to calculate $\lambda_{\pi}$ in each pixel as

$$
\lambda_{\pi}=\min \left\{\lambda_{\Omega}, \lambda\right\}
$$

with

$$
\lambda_{\Omega}:=\frac{-\ln (\xi)+\ln (\alpha)}{f\left(\nabla I_{1}\right)},
$$


TABLE I

SUMMARY OF REGULARIZATION STRATEGIES. I, THE IDENTITY MATRIX. $g(\cdot)$ A DECREASING FUNCTION. $\mathbf{n}=\frac{\nabla I_{1}}{\left|\nabla I_{1}\right|}$ AND $\mathbf{n}^{\perp}$ ITS ORTHONORMAL VECTOR. ROBUSTIFICATION CAN BE APPLIED to 'all' OR ONLY $\mathbf{n}, \mathbf{n}^{\perp}$ DIRECTIONS. $\mathcal{R}$ IS THE REGULARISER.

\begin{tabular}{|l|c|c|c|c|}
\hline Method & $\begin{array}{c}\text { Rotationally } \\
\text { Invariant }\end{array}$ & Robustification & Norm & $\mathcal{R}\left(\nabla I_{1}, \nabla u, \nabla v\right)$ \\
\hline \hline Horn-Schunck [1] & $\checkmark$ & - & $L^{2}$ & $|\nabla u|^{2}+|\nabla v|^{2}$ \\
Zach et al. [11] & - & - & $L^{1}$ & $|\nabla u|+|\nabla v|$ \\
Alvarez et al. [12] & $\checkmark$ & - & $L^{2}$ & $g\left(\left|\nabla I_{1}\right|\right) \cdot\left(|\nabla u|^{2}+|\nabla v|^{2}\right)$ \\
Brox et al. [10] & $\checkmark$ & all & $L^{1}$ & $\Psi\left(|\nabla u|^{2}+|\nabla v|^{2}\right)$ \\
Xu et al. [29] & - & all & $L^{1}$ & $g\left(\left|\nabla I_{1}\right|\right) \cdot(|\nabla u|+|\nabla v|)$ \\
\hline \hline Nagel-Enkelmann [2] & $\checkmark$ & - & $L^{2}$ & $\Psi u^{T} \mathcal{Z} \nabla u^{T}+\nabla v^{T} \mathcal{Z} \nabla v^{T}, \mathcal{Z}^{2}=\frac{\nabla I_{1}^{\perp} \nabla I_{1}^{\perp T}+\beta^{2} \mathbf{I}}{\left|\nabla I_{1}\right|^{2}+2 \beta^{2}}$ \\
Sánchez et al. [38] & $\checkmark$ & all & $L^{1}$ & $\left.\Psi u^{T} \mathcal{Z} \nabla u^{T}+\nabla v^{T} \mathcal{Z} \nabla v^{T}\right)$ \\
Werlberger et al. [5] & - & all & Huber- $L^{1}$ & $\left|\left(g\left(\left|\nabla I_{1}\right|^{2}\right) \mathbf{n n} \mathbf{n}^{T}+\mathbf{n}^{\perp} \mathbf{n}^{\perp T}\right) \nabla u\right|_{\epsilon}+\left|\left(g\left(\left|\nabla I_{1}\right|^{2}\right) \mathbf{n} \mathbf{n}^{T}+\mathbf{n}^{\perp} \mathbf{n}^{\perp T}\right) \nabla v\right|_{\epsilon}$ \\
Zimmer et al. [6] & $\checkmark$ & $\mathbf{n}$ & $L^{1}$ & $\Psi\left(u_{r_{1}}^{2}+v_{r_{1}}^{2}\right)+\left(u_{r_{2}}^{2}+v_{r_{2}}^{2}\right),\left(r_{1}, r_{2}\right)$ eigenvalues of a structure tensor \\
\hline
\end{tabular}

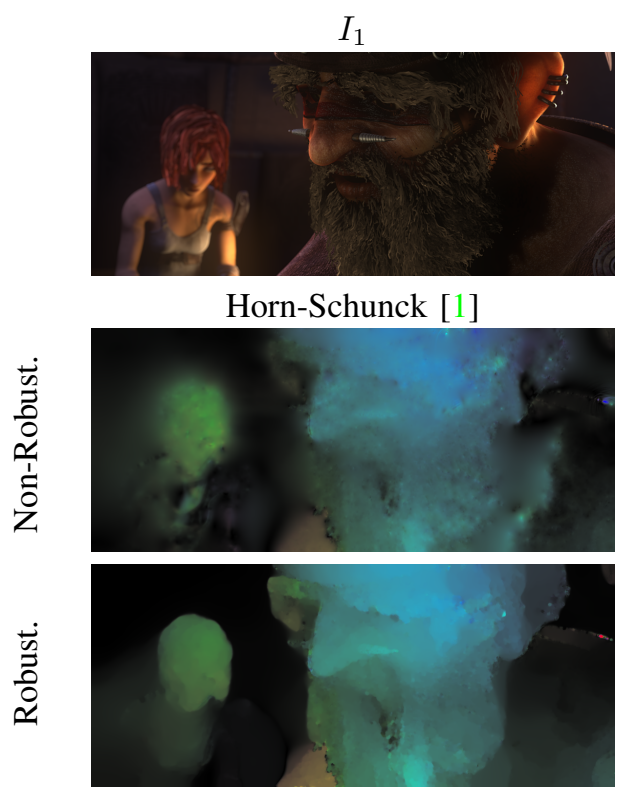

Brox et al. [10]

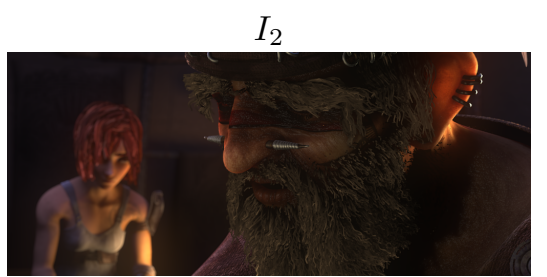

Nagel-Enkelmann [2]
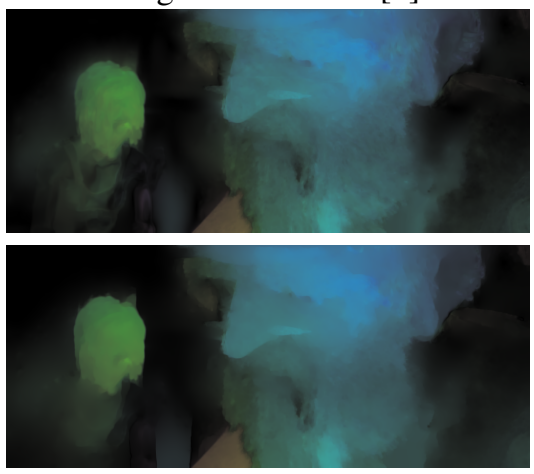

$R A D T$



Alvarez et al. [3]
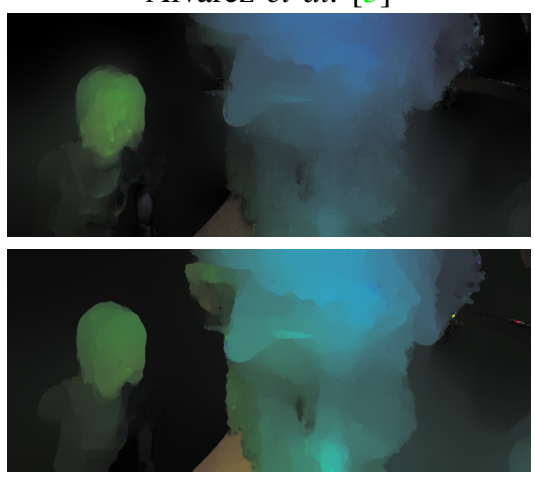

DF [17]

Fig. 2. Top row: two frames of the Shaman 2 sequence and its ground truth. Middle row: the flow fields obtained with the Horn-Schunck, Nagel-Enkelmann and Alvarez methods. Bottom row: the solutions given by the Brox, a robust diffusion tensor and $D F$ methods. We see how robustification techniques reduce the influence of outliers and their combination with decreasing functions allows to obtain piecewise-smooth flow fields.

where $f\left(\nabla I_{1}\right)$ is a statistical function of the image gradients. One possible alternative is

$$
f_{1}\left(\nabla I_{1}\right)=\tau \cdot \max _{\mathbf{x} \in \Omega}\left\{\left|\nabla I_{1}(\mathbf{x})\right|\right\}
$$

with $\tau \in(0,1]$. Another alternative is to rely on the histogram of the gradient as

$$
f_{2}\left(\nabla I_{1}\right)=\left|\nabla I_{1}\left(\mathbf{x}^{\prime}\right)\right|
$$

with

$$
\tau:=\frac{\sum_{\mathbf{x}:=0}^{\mathbf{x}^{\prime}} \mathcal{H}\left(\left|\nabla I_{1}(\mathbf{x})\right|\right)}{\sum_{\mathbf{x} \in \Omega} \mathcal{H}\left(\left|\nabla I_{1}(\mathbf{x})\right|\right)},
$$

with $\mathcal{H}\left(\left|\nabla I_{1}(\mathbf{x})\right|\right)$ the histogram of the gradient and $\tau \in$ $(0,1]$.

In both cases, $\tau$ determines the conservative behavior of $\lambda_{\Omega}$. The first alternative, with $\tau:=1$, was used in [19]. It prevents the occurrence of instabilities, however, it restricts the detection of less prominent motion contours. Varying the value of $\tau$ allows to detect more discontinuities. Nevertheless, it is difficult to fix a good parameter for all types of sequences, as we can see on the left graphic in Fig. 3.

For the second alternative, we see on the right graphic in Fig. 3 that the evolution with respect to $\tau$ converges and the best results are bounded between 0.9 and 0.95 for all the 


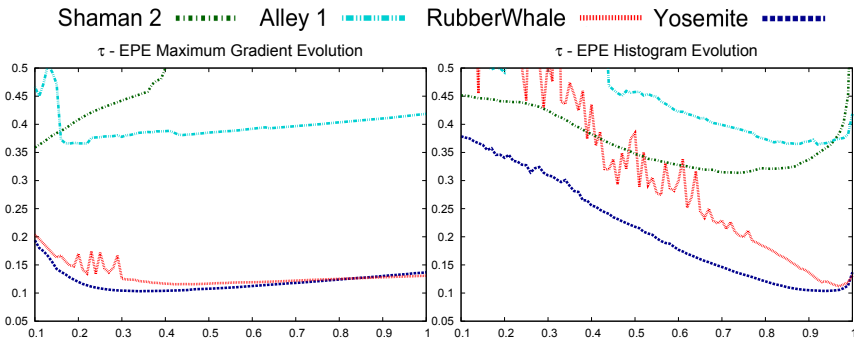

Fig. 3. Average End Point Error (EPE) evolution with respect to $\tau$ using the two strategies proposed for $\lambda$ estimation, $f_{1}\left(\nabla I_{1}\right)$ and $f_{2}\left(\nabla I_{1}\right)$. We see the evolution of the EPE error for several sequences. For the first proposal, we see that the results are very stable. However, the second proposal is more interesting, since the best $\tau$ is bounded between 0.9 and 0.95 .

sequences. In this sense, $\tau$ can be considered as a constant and we fix its value to $\tau:=0.94$ in our experiments. Since this strategy is more robust, due to the use of the histogram, and less dependent on $\tau$, this is the best of the two alternatives. Therefore, we will use $f_{2}\left(\nabla I_{1}\right)$ in the experiments.

Other approaches can be used, such as statistics based on the the average or median values of the image gradients.

We must note that the value of the regularization parameter $\alpha$ is influenced by the decreasing function and, therefore, by the $\lambda$ parameter. Thus, the automatic estimation of $\lambda$ offers an indirect benefit, which is a joint normalization with the smoothness weight. In this sense, $\lambda$ is adapted to the $\alpha$ value: when it is small, the value of $\lambda$ will be also small. Even, if $\alpha$ is smaller than 1 , the value of $\lambda$ will become negative and the exponential will turn increasing, which avoids canceling the regularization term.

5) Robust anisotropic diffusion tensor (RADT):

$$
\begin{aligned}
\mathcal{R}\left(\nabla I_{1}, \nabla u, \nabla v\right) & :=\Phi\left(\left(\mathbf{n}^{T} \nabla u\right)^{2}+\left(\mathbf{n}^{T} \nabla v\right)^{2}\right) \\
& +\left(\mathbf{n}^{\perp} \nabla u\right)^{2}+\left(\mathbf{n}^{\perp} \nabla v\right)^{2}
\end{aligned}
$$

with $\Phi\left(s^{2}\right)=\frac{1}{\lambda^{2}} \log \left(1+\lambda^{2} s^{2}\right)$.

This diffusion tensor resembles a robustification variant of the Nagel-Enkelmann operator, with decreasing functions to mitigate the diffusion across the boundaries of the objects. The diffusion is performed unconditionally along the isocontours while in the gradient direction it is modulated by a robustification function.

The behavior of the diffusion tensors is depicted in Fig. 2 . We see how robustification techniques reduce the influence of noise and allow to obtain piecewise-smooth flow fields.

The solution given by the Nagel-Enkelmann method improves the Horn-Schunck solution at the edges, as can be seen in the head of the girl.

Both, Brox and Nagel-Enkelmann methods, obtain piecewise-smooth motion fields. However, the NagelEnkelmann method cannot preserve discontinuities when the gradient of the image is small, as we see in the beard and the eyebrow. The Brox method detects both of them, although it does not distinguish the hair tufts. The Total Variation tends to smooth the flow destroying details. It also creates rounded shapes in high curvature boundaries.
The flow fields obtained by the Alvarez and $D F$ methods are very similar. The most significant difference between them is the magnitude of the flow, especially in the eyebrow and beard. Furthermore, the robustification has allowed to detect discontinuities with small image gradients. In the $D F$ approach, we see sharp discontinuities but small artifacts close to the old man ear.

The solution obtained by $R A D T$ identifies the main objects in the scene. However, it suffers the limitations of NagelEnkelmann and the motion edges are not so clear as in the Brox or $D F$ flow fields. The discontinuities are not clearly preserved due to the small gradients.

\section{B. Minimizing the energy functional}

The minimum of the energy functional (1) can be found by solving the associated Euler-Lagrange equations. The data term is given in (2) and the smoothing term is adapted to the diffusion tensors presented in table II. Then, the EulerLagrange equations are given by

$$
\begin{aligned}
0= & \Psi_{B}^{\prime} \cdot\left(\sum_{c=1}^{C} I_{2}^{c}(\mathbf{x}+\mathbf{w})-I_{1}^{c}(\mathbf{x})\right) \cdot I_{2, x}^{c}(\mathbf{x}+\mathbf{w}) \\
& +\gamma \Psi_{G}^{\prime} \cdot\left(\sum_{c=1}^{C}\left(I_{2, x}^{c}(\mathbf{x}+\mathbf{w})-I_{1, x}^{c}(\mathbf{x})\right) \cdot I_{2, x x}^{c}(\mathbf{x}+\mathbf{w})\right. \\
& \left.+\sum_{c=1}^{C}\left(I_{2, y}^{c}(\mathbf{x}+\mathbf{w})-I_{1, y}^{c}(\mathbf{x})\right) \cdot I_{2, x y}^{c}(\mathbf{x}+\mathbf{w})\right) \\
& -\alpha \operatorname{div}\left(\mathbf{D}\left(\nabla I_{1}, \nabla u, \nabla v\right) \cdot \nabla u\right), \\
0= & \Psi_{B}^{\prime} \cdot\left(\sum_{c=1}^{C} I_{2}^{c}(\mathbf{x}+\mathbf{w})-I_{1}^{c}(\mathbf{x})\right) \cdot I_{2, y}^{c}(\mathbf{x}+\mathbf{w}) \\
& +\gamma \Psi_{G}^{\prime} \cdot\left(\sum_{c=1}^{C}\left(I_{2, x}^{c}(\mathbf{x}+\mathbf{w})-I_{1, x}^{c}(\mathbf{x})\right) \cdot I_{2, x y}^{c}(\mathbf{x}+\mathbf{w})\right. \\
& \left.+\sum_{c=1}^{C}\left(I_{2, y}^{c}(\mathbf{x}+\mathbf{w})-I_{1, y}^{c}(\mathbf{x})\right) \cdot I_{2, y y}^{c}(\mathbf{x}+\mathbf{w})\right) \\
& -\alpha \operatorname{div}\left(\mathbf{D}\left(\nabla I_{1}, \nabla u, \nabla v\right) \cdot \nabla v\right),
\end{aligned}
$$

with $\Psi^{\prime}\left(s^{2}\right)=\frac{1}{2 \sqrt{s^{2}+\epsilon^{2}}}$. In order to simplify these equations, we have used the following notation:

$$
\begin{aligned}
& \Psi_{B}^{\prime}:=\Psi^{\prime}\left(\sum_{c=1}^{C}\left(I_{2}^{c}(\mathbf{x}+\mathbf{w})-I_{1}^{c}(\mathbf{x})\right)^{2}\right), \\
& \Psi_{G}^{\prime}:=\Psi^{\prime}\left(\sum_{c=1}^{C}\left|\nabla I_{2}^{c}(\mathbf{x}+\mathbf{w})-\nabla I_{1}^{c}(\mathbf{x})\right|^{2}\right),
\end{aligned}
$$

and $\mathbf{D}\left(\nabla I_{1}, \nabla u, \nabla v\right)$ the diffusion tensors shown in Table II.

In order to solve this system, we discretize the equations using centered finite differences. Then, the system of equations is solved by means of an iterative approximation, such as the SOR method. Due to the nonlinear nature of these formulas, the resolution of these equations requires two fixed point iterations, in order to converge to a steady state. The warpings of $I_{2}$ are approximated using Taylor expansions.

These equations are embedded in a multiscale strategy that allows to recover large displacements. Starting from the 
TABLE II

THE REgUlARISERS ANALYZED AND THEIR DIFFUSION TENSORS, D. I REPRESENTS THE IDENTITY MATRIX. $\mathbf{n}=\frac{\nabla I_{1}}{\left|\nabla I_{1}\right|}$ AND $\mathbf{n}^{\perp}$ ITS ORTHONORMAL VECTOR. $\Psi\left(s^{2}\right)=\sqrt{s^{2}+\epsilon^{2}}, \Psi^{\prime}\left(s^{2}\right)=\frac{1}{2 \sqrt{s^{2}+\epsilon^{2}}}, \Phi\left(s^{2}\right)=\frac{1}{\lambda^{2}} \log \left(1+\lambda^{2} s^{2}\right)$ AND $\Phi^{\prime}\left(s^{2}\right)=\frac{1}{1+\lambda^{2} \cdot s^{2}}$

\begin{tabular}{|c|c|c|c|}
\hline Method & $\mathcal{Z}$ & $\mathcal{R}\left(\nabla I_{1}, \nabla u, \nabla v\right)$ & $\mathbf{D}\left(\nabla I_{1}, \nabla u, \nabla v\right)$ \\
\hline $\begin{array}{l}\text { Brox } \\
D F \\
D F-\beta \\
\text { DF-Auto }\end{array}$ & $\begin{array}{c}1 \\
e^{-\lambda\left|\nabla I_{1}\right|} \\
e^{-\lambda\left|\nabla I_{1}\right|}+\beta \\
e^{-\lambda_{\pi}\left|\nabla I_{1}\right|}\end{array}$ & $\Psi\left(\mathcal{Z} \cdot\left(|\nabla u|^{2}+|\nabla v|^{2}\right)\right)$ & $\mathcal{Z} \cdot \Psi^{\prime}\left(\mathcal{Z} \cdot\left(|\nabla u|^{2}+|\nabla v|^{2}\right)\right) \cdot \mathbf{I}$ \\
\hline$R A D T$ & - & $\Phi\left(\left(\mathbf{n}^{T} \nabla u\right)^{2}+\left(\mathbf{n}^{T} \nabla v\right)^{2}\right)+\left(\mathbf{n}^{\perp} \nabla u\right)^{2}+\left(\mathbf{n}^{\perp} \nabla v\right)^{2}$ & $\Phi^{\prime}\left(\left(\mathbf{n}^{T} \nabla u\right)^{2}+\left(\mathbf{n}^{T} \nabla v\right)^{2}\right)+\mathbf{n}^{\perp} \mathbf{n}^{\perp T}$ \\
\hline
\end{tabular}

coarsest scales, we obtain a solution to the above system, and then upgrade the value of the optical flow for the next finer scale. We use motion increments, $\mathbf{w}^{k+1}=\mathbf{w}^{k}+\mathbf{d w}^{k}$, so that, in each scale, we compute each increment, $\mathbf{d w}^{k}$, and the final optical flow is obtained as an accumulative value for all increments. Details on the discretization of this scheme are given in [10] or, more extensively, in [30].

\section{EXPERIMENTAL RESULTS}

In order to demonstrate the hypotheses of our work, we first analyzed the stability properties of each method. For this, we selected representative sequences from several datasets and studied the behavior of the methods with respect to the discontinuity parameter $\lambda$ (Sect. IV-A).

Then, we compared the methods and showed some visual results where the differences and features of each one are clearly noticeable (Sect. IV-B). This helps understand that the effect of instabilities is not accidental but it appears very frequently.

Finally, in Sect. IV-C, we carried out an extensive numerical evaluation of the methods using the test and evaluation sequences from the MPI-Sintel [39] and Middlebury [40] benchmark databases, which allows us to show the results in a more general setting.

The parameters were set according to [19] except for $\alpha, \gamma$ and $\lambda$ that were adapted in each experiment. The motion fields are represented using the color scheme shown in Fig. 1. The color represents the motion orientation while the intensity its magnitude.

\section{A. Analysis of the methods stability}

In this section, we compare the average End-Point Error (EPE) evolution with respect to $\lambda$. Figure 4 shows the results for several sequences from the MPI-Sintel and Middlebury datasets.

In these graphics, the error evolution of the $D F$ method is depicted with a blue line. We observe that it consistently improves the Brox approach $(\lambda:=0)$. The improvement is remarkable in the graphics of Shaman 2, Bamboo 1, Urban 3 or RubberWhale.

However, we see that the $D F$ method is very unstable. The errors increase very fast once the best solution is achieved.
From this point, the blobs seen in Fig. 1 begin to appear. For Shaman 2, this strategy provides more stable results for a large range of $\lambda$ values. This sequence is very interesting because it presents very bright and dark areas. This circumstance allows increasing the parameter and still yielding good solutions.

In contrast, the $D F-\beta$ approach (violet line) is much more stable, presenting a smoother evolution. This is due to the small constant $(\beta)$ that prevents the regularization to get canceled. In this sense, even with an extreme parameter, its effects over the optical flow are quite similar. For most of the sequences, it improves the results when $D F$ strongly deteriorates the solutions.

On the other hand, the DF-Auto proposal (red line) offers results close to the best solutions obtained with the other exponential schemes. This method controls the diffusion locally for every pixel. This provides an efficient parameter that adapts to homogeneous and discontinuous regions.

$D F$ and $D F-\beta$ use a unique parameter value for the whole image. Thus, the control of the diffusion only depends on the gradient strength. Interestingly, the DF-Auto strategy adjusts $\lambda$ for being less incisive in areas with low gradients and strengthen the decreasing function in zones with strong variations.

Finally, the $R A D T$ error evolution is depicted with a green line. In this case, we have used a range for $\lambda \in[0,50]$ and we have normalized the results between $[0,1]$ to fit into the graphics. Note that, when $\lambda:=0$, the regularization becomes the Horn-Schunck approach. This is the reason why the starting error is usually bigger than the other strategies. However, this scheme is the most stable: the best errors remain almost constant for large values of $\lambda$. This is reasonable, because, unlike the other schemes, there always exists a regularization along the isocontours of the objects. For a sufficiently large value of $\lambda$, the robustification function cancels the regularization across the borders of the object. Then, the results only depend on the isocontour smoothing, which is always the same regardless of $\lambda$.

From these results, we may conclude the following: The basic $D F$ scheme turns unstable very fast from a given $\lambda$ value. The range of interest values is very small in practice. The $D F-\beta$ provides better stability results in general at the same time that it preserves the accuracy of the basic method. In some cases, it yields improved results. Due to its simplicity, 

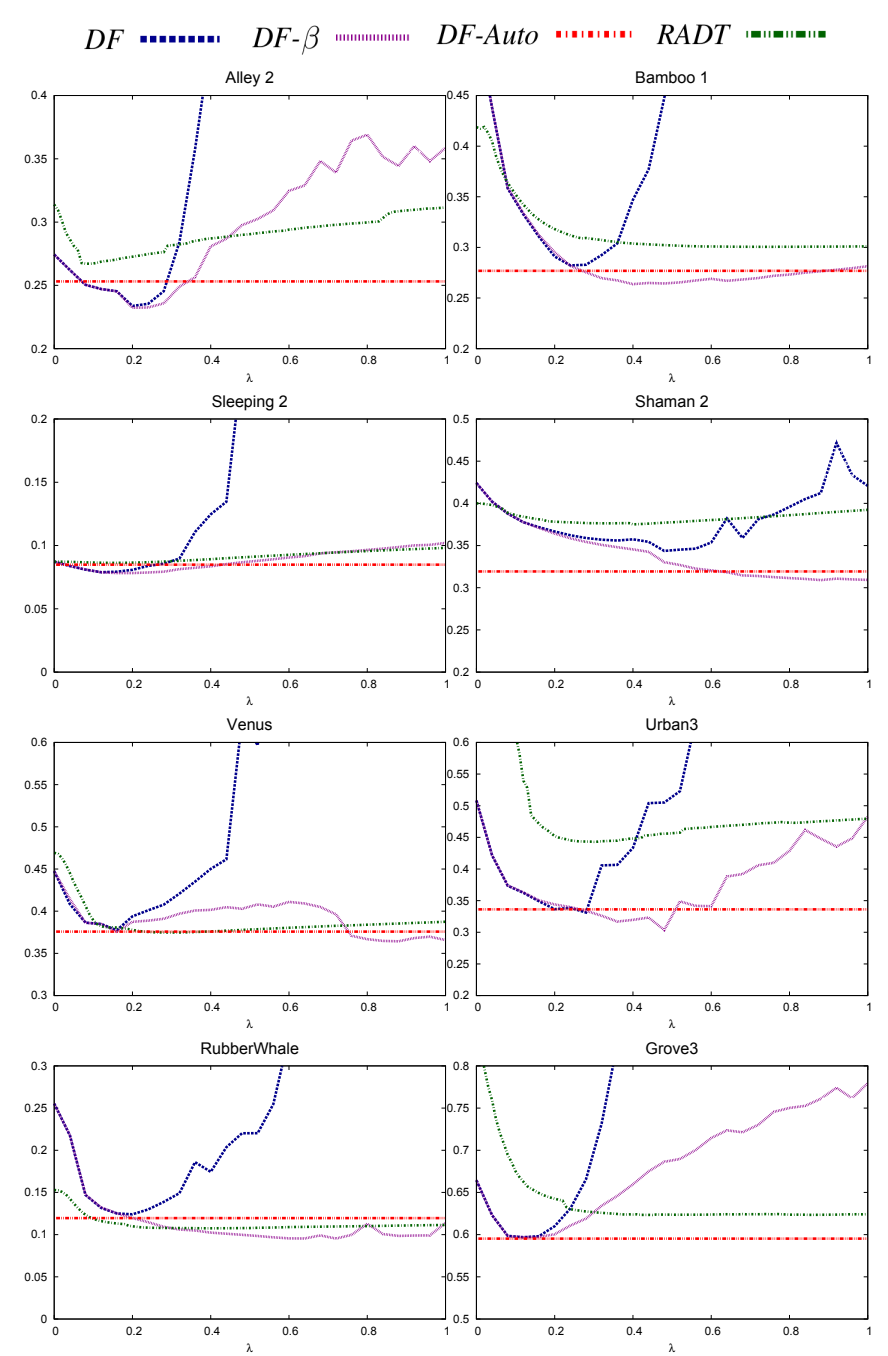

Fig. 4. Average End Point Error (EPE) evolution with respect to $\lambda$ for $M P I-$ Sintel and Middlebury sequences. The use of decreasing functions improves the solutions obtained with the Brox approach $(\lambda:=0)$. In general, the $D F$ method is very sensitive to $\lambda$, whereas the $D F-\beta$ and $R A D T$ approaches are more stable, requiring a minimum setup. The DF-Auto method is close to the best result for most of the sequences.

this approach is preferable with respect to the $D F$ technique. The DF-Auto is very reliable and, in most cases, it provides results which are near the best solutions. On the other hand, the $R A D T$ method is very stable and does not produce instabilities in general. This is a very interesting feature, however, the accuracy is typically worse. The use of more advanced techniques, like in [6], may turn this method more competitive.

\section{B. Comparison of the methods}

The first row of Figs. 5, 6, 7 and 8 show several sequences and their EPE graphics with respect to $\lambda$. The second and third rows show the different flow fields and the ground truth.

We observe that the Brox method does not usually detect correct motion contours. The flow discontinuities are in general not aligned with the object contours, as we can clearly see in the Rectangles test. In this case, the method cannot distinguish between the two rectangles. We also observe that it has problems with the leafs in Grove2 and the hair tufts of
Original Image
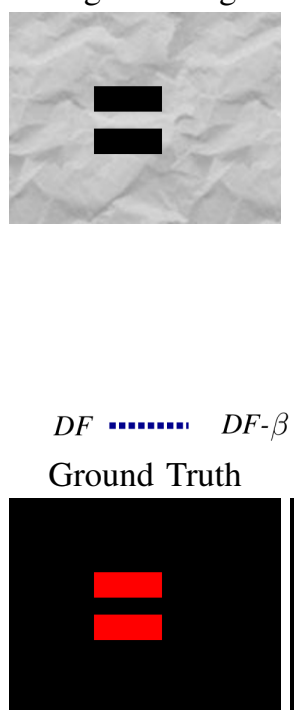

$D F$
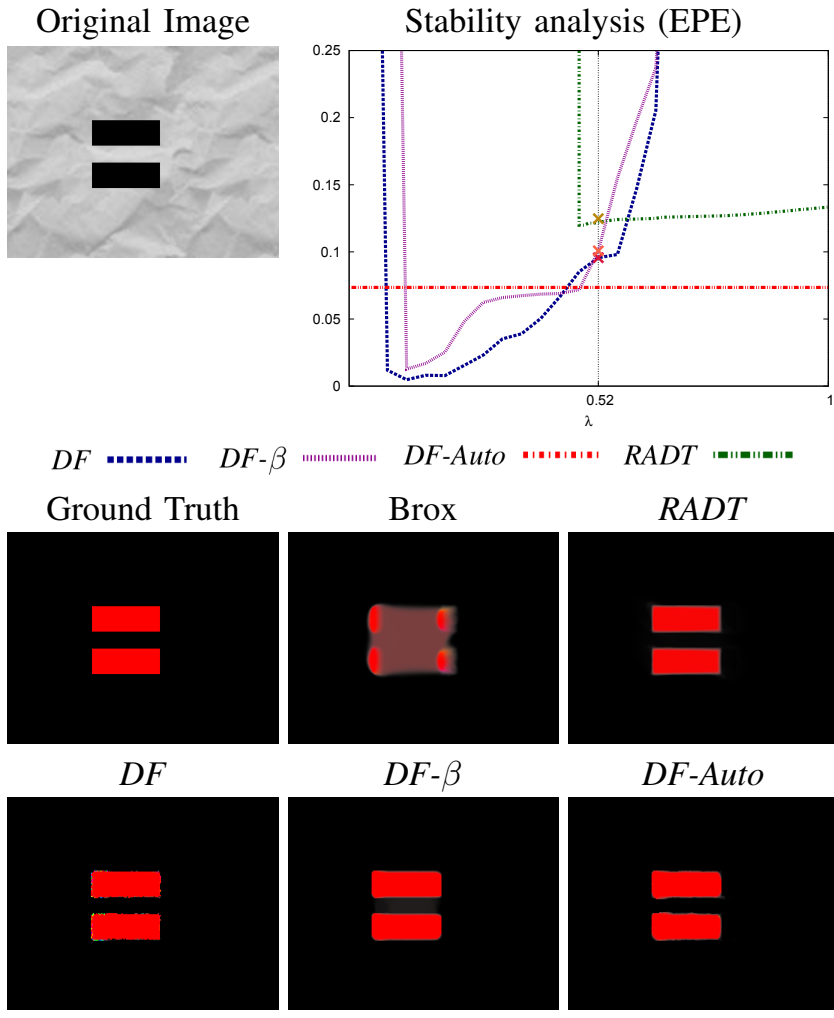

$\lambda$

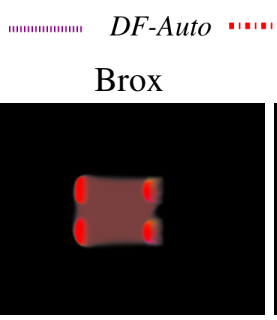

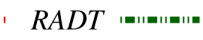
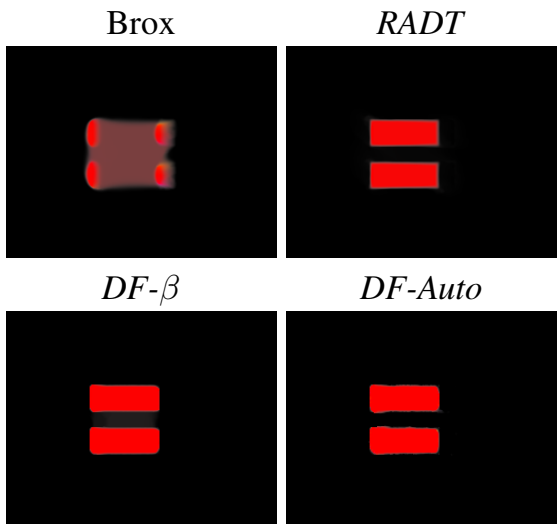

DF-Auto

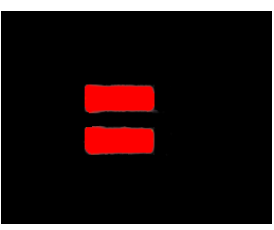

Fig. 5. First row: The Rectangles sequence and its EPE graphic with respect to $\lambda$. Second row: the ground truth and the solutions obtained with Brox and $R A D T$ methods, respectively. Third row: $D F, D F-\beta$ and $D F$-Auto solutions. The $D F$ detects the discontinuities but introduces some instabilities. The $D F$ $\beta$ and $D F$-Auto variants eliminate these instabilities but create slightly rounded flows. The RADT approach creates straight edges but these are not so sharp.

the woman in Alley 1.

This situation is ameliorated with the other schemes, principally in Fig. 5, where the other approaches achieve very accurate edges.

These four figures are a good example of the high parametric dependence of the $D F$ method. We observe that numerous flow instabilities appear with this approach, while the others provide a reasonable quality. In fact, the solution of our first proposal is quite similar to the $D F$ method, but with an important reduction of blobs.

For comparing the stability of $D F, D F-\beta$ and $R A D T$, we have intentionally taken a value of $\lambda$ which is slightly bigger than its optimal value. This is represented by the vertical lines in the graphics and the $X$ marks. Note that these points do not always appear for the $D F$ graphic because the error is normally too big.

On the other hand, the RADT scheme offers good solutions. Nevertheless, we observe that the diffusion is not completely stopped at the object contours, especially at the skyline in the Yosemite sequence; Fig. 6. This phenomenon is similar to the Shaman 2 sequence, Fig. 2, where the small gradients do not allow to steer the diffusion process conveniently. We can also see a good result in Fig. 8. The method correctly detects the woman arm and the apple in her hand.

Figure 7 offers an interesting issue that we must consider: According to the graphic, the gain with respect to the Brox er- 



Fig. 6. First row: Yosemite sequence and its EPE graphic with respect to $\lambda$. Second row: the ground truth and the solutions obtained with Brox and $R A D T$ methods, respectively. Third row: $D F, D F-\beta$ and $D F$-Auto solutions. The flow field obtained by Brox visually resembles the ground truth, except in the skyline and the mountain discontinuities. The textures in the mountains create slightly segmented flows in the other methods. $D F$ also introduces instabilities in high gradient regions. $D F-\beta$ and $D F$-Auto eliminate these instabilities. $R A D T$ provides a good flow except in the skyline.

ror is not remarkable using the other approaches. Nevertheless, they show a better contour definition but with some small instabilities. These failures worsen the average error of the whole flow. For this reason, it is possible that a motion field, with better edges, may present a similar error.

From these experiments, we may conclude that this type of discontinuity-preserving strategies offers a precise definition of motion contours only if the parameters are correctly chosen. Otherwise, this strategy could provide poorest results than the Brox approach and may turn unstable. Interestingly, our two proposals yield good optical flows with more stable solutions for a large range of parameters. These two features make both proposals very interesting for real applications.

In summary, we observe the same behavior as in the previous section. From the Rectangles sequence we can appreciate the limitations of the Brox method and the benefits of using the other strategies. The motion contours are much better preserved even though the EPE errors are not so different with respect to Brox. The graphics show the instability problems of the $D F$ method and we can clearly see the blobs in the flow images. This means that there is a correlation between the choice of $\lambda$, the appearance of instabilities and the increase of EPE errors. The RADT method preserves good motion discontinuities but the flow field is smoother than $D F-\beta$ and DF-Auto. However, the latter still introduce some instabilities
Original Image
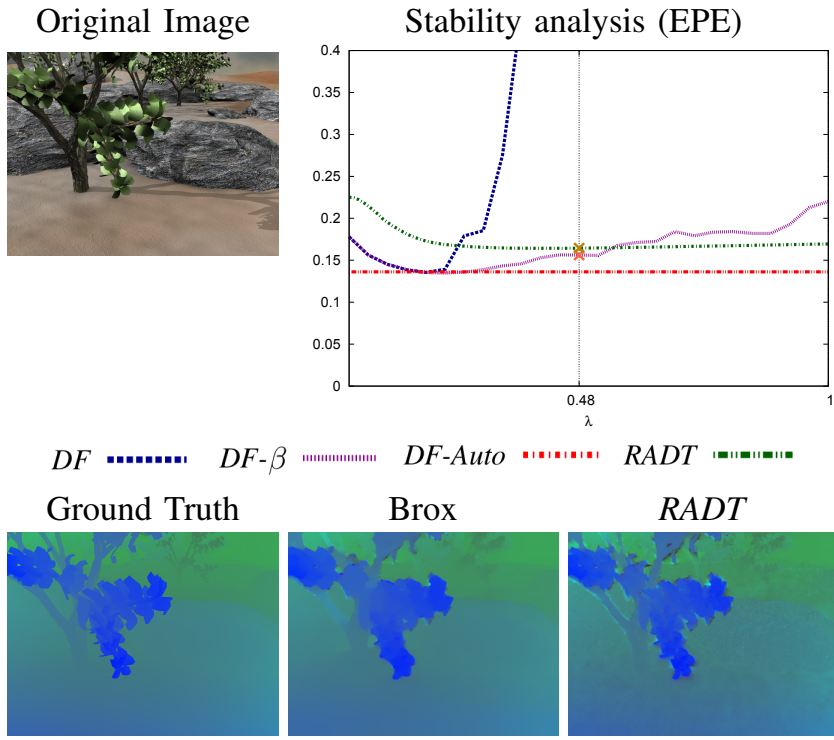

$D F$

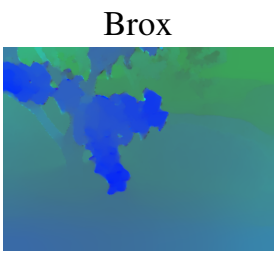

$D F-\beta$
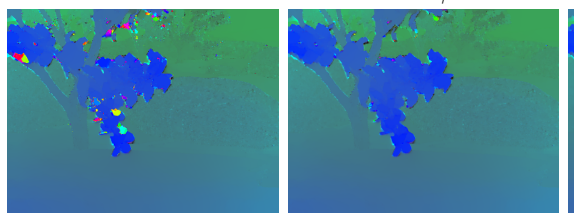

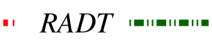

Fig. 7. First row: Grove2 sequence and its EPE graphic with respect to $\lambda$. Second row: the ground truth and the solutions obtained with Brox and $R A D T$ methods, respectively. Third row: $D F, D F-\beta$ and $D F$-Auto solutions. In constrast to the Brox method, the discontinuity-preserving strategies offer an accurate flow at motion edges. In general, the behavior is similar to Yosemite in Fig. 6.
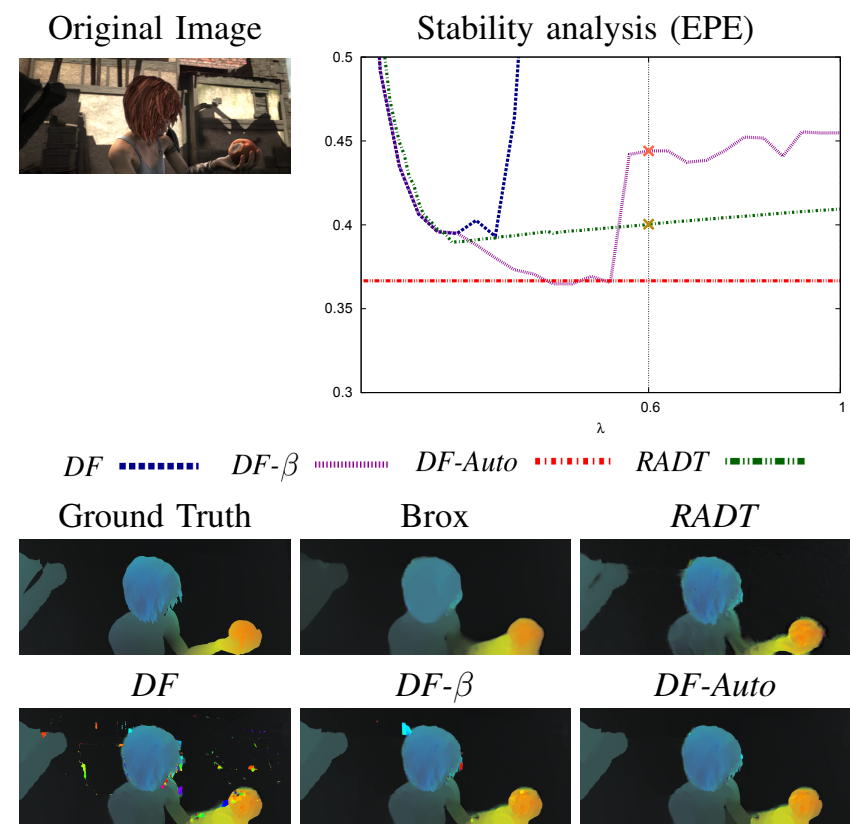

DF-Auto

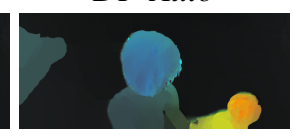

Fig. 8. First row: Alley 1 sequence and its EPE graphic with respect to $\lambda$. Second row: the ground truth and the solutions obtained with Brox and $R A D T$ methods, respectively. Third row: $D F, D F-\beta$ and $D F$-Auto solutions. The main differences are located in the woman arm and in the apple.

around the contours, probably at occluded regions, which are not present at $R A D T$. 
TABLE III

AVERAGe EPE For the TEST SEQUENCES OF Middlebury AND Sintel TRAINING DATASETS

\begin{tabular}{|l|c|c|c|c|}
\cline { 2 - 5 } \multicolumn{1}{c|}{} & $\lambda$ & Middlebury & Sintel-final & Sintel-clean \\
\hline IPOL-Brox $[30]$ & - & $\mathbf{0 . 3 2 6}$ & $\mathbf{7 . 8 2 3}$ & $\mathbf{5 . 0 6 2}$ \\
\hline$D F$ & 0.1 & $\mathbf{0 . 2 9 4}$ & $\mathbf{7 . 9 9 5}$ & $\mathbf{5 . 1 9 5}$ \\
$D F$ & 0.3 & 0.326 & 9.048 & 6.312 \\
$D F$ & 0.5 & 0.559 & 10.467 & 7.903 \\
\hline$D F-\beta$ & 0.1 & 0.295 & $\mathbf{8 . 0 3 5}$ & $\mathbf{5 . 1 9 0}$ \\
$D F-\beta$ & 0.3 & $\mathbf{0 . 2 9 2}$ & 8.643 & 5.823 \\
$D F-\beta$ & 0.5 & 0.312 & 9.194 & 6.405 \\
\hline$D F-$ Auto & - & $\mathbf{0 . 2 9 8}$ & $\mathbf{8 . 4 2 9}$ & $\mathbf{5 . 9 2 9}$ \\
\hline
\end{tabular}

TABLE IV

Best Average EPE for the EVALUATION SEQUences of Middlebury DATASET

\begin{tabular}{|l|c|c|}
\cline { 2 - 3 } \multicolumn{1}{c|}{} & Average EPE & Rank \\
\hline OFH [6] & 0.360 & 41.0 \\
EpicFlow [41] & 0.3925 & 54.3 \\
DF- $\beta$ & 0.4450 & 68.7 \\
DF-Auto & 0.4563 & 66.9 \\
Brox [10] & 0.5013 & 68.3 \\
TV-L1-improved [42] & 0.5438 & 62.7 \\
LDOF [43] & 0.5613 & 79.2 \\
\hline
\end{tabular}

\section{Numerical results}

Next, we present numerical results using the complete datasets of Middlebury and Sintel. Table III shows the average EPE, using different $\lambda$ values for $D F$ and $D F-\beta$. We observe that the best average error for Middlebury is achieved by $D F$ $\beta$ with $\lambda=0.3$. Interestingly, the error does not strongly varied for the other $\lambda$ values. On the other hand, $D F$ attains its best result at $\lambda=0.1$. However, the error considerably increases for the other values. DF-Auto scheme obtains a good solution close to the best error. This behavior is similar for the exponential approaches using the Sintel dataset. However, Brox presents better numerical errors despite of its worst behaviour at motion contours (see Fig. 10).

In Table IV, we compare our strategies with some of the methods presented in the Middlebury ranking. We have chosen the techniques that are somehow related to our approaches. However, note that these are usually much more complex and it is difficult to draw clear conclusions. We see that the results of $D F-\beta$ and DF-Auto are better than Brox. In Figure 9, we show the computed flows for three sequences. We observe that the DF-Auto method preserves the contours of the moving objects, even better than $O F H$ and EpicFlow in many cases. However, we see that the errors at the occlusions are bigger. This is reasonable because the method does not include any mechanism to deal with this problem.

In Tables V and VI, we show the errors for each sequence of the test dataset of Sintel, which are published on the web page. We compare with other related methods, such as EpicFlow, LDOF, Horn and Schunck and IPOL-Brox. We observe that, as in the previous table, the methods achieve similar results. The proposed methods present competitive results despite of their simplicity. We also notice that the $D F$ scheme required much more training to obtain a suitable configuration, whereas $D F-\beta$ and DF-Auto did not.

In Tables VII and VIII, we show the global errors. In
TABLE V

Results By SEQUenCE ON THE MPI Sintel Clean TEST SUBSET

\begin{tabular}{|l|l|l|l|l|l|l|l|}
\cline { 2 - 7 } \multicolumn{1}{c|}{} & $\begin{array}{l}\text { Epic } \\
\text { Flow }\end{array}$ & LDOF & HS & $\begin{array}{l}\text { IPOL- } \\
\text { Brox }\end{array}$ & DF & DF- $\beta$ & $\begin{array}{l}\text { DF- } \\
\text { Auto }\end{array}$ \\
\hline P. Market 3 & 0.807 & 1.177 & 1.450 & 1.191 & 1.263 & 1.263 & 1.468 \\
P. Shaman 1 & 0.501 & 1.612 & 1.895 & 1.857 & 1.649 & 1.651 & 1.581 \\
Ambush 1 & 9.037 & 34.70 & 32.97 & 20.63 & 22.65 & 22.69 & 33.44 \\
Ambush 3 & 5.657 & 8.960 & 10.60 & 9.233 & 9.391 & 9.325 & 9.880 \\
Bamboo 3 & 1.030 & 1.036 & 1.339 & 1.106 & 1.237 & 1.228 & 1.344 \\
Cave 3 & 5.075 & 7.550 & 12.41 & 8.635 & 8.923 & 8.929 & 11.40 \\
Market 1 & 2.211 & 3.233 & 4.650 & 4.456 & 4.396 & 4.392 & 5.820 \\
Market 4 & 22.05 & 38.43 & 42.94 & 37.99 & 38.18 & 38.06 & 40.79 \\
Mountain 2 & 0.225 & 1.179 & 0.233 & 1.025 & 1.028 & 1.028 & 1.094 \\
Temple 1 & 0.818 & 1.460 & 2.056 & 1.484 & 1.397 & 1.395 & 1.443 \\
Tiger & 0.630 & 1.254 & 1.064 & 0.954 & 1.101 & 1.097 & 1.134 \\
Wall & 4.330 & 5.372 & 6.950 & 6.554 & 6.419 & 6.418 & 6.375 \\
\hline
\end{tabular}

TABLE VI

RESUlts By SEQUENCE ON THE MPI Sintel Final TEST SUBSET

\begin{tabular}{|l|l|l|l|l|l|l|l|}
\cline { 2 - 8 } \multicolumn{1}{c|}{} & $\begin{array}{l}\text { Epic } \\
\text { Flow }\end{array}$ & LDOF & HS & $\begin{array}{l}\text { IPOL- } \\
\text { Brox }\end{array}$ & DF & DF- $\beta$ & $\begin{array}{l}\text { DF- } \\
\text { Auto }\end{array}$ \\
\hline P. Market 3 & 1.290 & 2.832 & 2.118 & 2.057 & 2.003 & 2.002 & 2.080 \\
P. Shaman 1 & 0.641 & 2.269 & 2.463 & 2.457 & 2.158 & 2.159 & 1.983 \\
Ambush 1 & 35.18 & 44.96 & 40.55 & 44.92 & 43.59 & 43.69 & 45.50 \\
Ambush 3 & 8.695 & 14.13 & 15.38 & 14.80 & 14.32 & 14.37 & 15.72 \\
Bamboo 3 & 1.087 & 1.107 & 1.419 & 1.196 & 1.355 & 1.353 & 1.483 \\
Cave 3 & 6.207 & 9.227 & 13.01 & 9.704 & 9.681 & 9.711 & 11.31 \\
Market 1 & 3.150 & 4.179 & 5.397 & 4.809 & 4.758 & 4.753 & 5.401 \\
Market 4 & 26.55 & 39.21 & 40.01 & 38.26 & 39.19 & 39.17 & 40.79 \\
Mountain 2 & 1.646 & 1.618 & 1.544 & 1.691 & 1.767 & 1.766 & 1.905 \\
Temple 1 & 1.295 & 1.606 & 2.069 & 1.741 & 1.846 & 1.841 & 1.909 \\
Tiger & 1.147 & 1.637 & 1.584 & 1.799 & 1.906 & 1.907 & 1.987 \\
Wall & 4.904 & 7.294 & 7.889 & 7.660 & 7.594 & 7.596 & 7.438 \\
\hline
\end{tabular}

the Clean dataset, the IPOL-Brox method provides slightly better results than the exponential strategies, whereas in the Final set occurs the opposite. However, the differences are not meaningful. DF-Auto results are slightly worse because of the errors in a few sequences with very large displacements and occlusions, like Ambush 1.

TABLE VII

REsults on the MPI Sintel Clean TeSt SUbSET

\begin{tabular}{|l|l|l|l|l|l|l|}
\cline { 2 - 7 } \multicolumn{1}{c|}{} & Rank & $\begin{array}{l}\text { EPE } \\
\text { all }\end{array}$ & $\begin{array}{l}\text { EPE } \\
\text { matched }\end{array}$ & $\begin{array}{l}\text { EPE un- } \\
\text { matched }\end{array}$ & $\begin{array}{l}\mathbf{d 0 -} \\
\mathbf{1 0}\end{array}$ & $\mathbf{s 4 0 +}$ \\
\hline EpicFlow [41] & 7 & 4.115 & 1.360 & 26.60 & 3.660 & 25.86 \\
IPOL-Brox [30] & 44 & 7.283 & 3.150 & 40.93 & 5.705 & 46.80 \\
DF- $\beta$ & 46 & 7.391 & 3.153 & 41.89 & 5.492 & 47.84 \\
DF & 47 & 7.406 & 3.164 & 41.94 & 5.504 & 47.95 \\
LDOF [43] & 48 & 7.563 & 3.432 & 41.17 & 5.353 & 51.70 \\
DF-Auto & 53 & 8.480 & 3.945 & 45.40 & 6.445 & 56.78 \\
HS [1] & 55 & 8.739 & 4.525 & 43.03 & 7.542 & 58.24 \\
AnisoHuber [5] & 61 & 12.64 & 7.983 & 50.47 & 10.457 & 77.84 \\
\hline
\end{tabular}

TABLE VIII

RESUlts ON THE MPI Sintel Final TEST SUBSET

\begin{tabular}{|l|l|l|l|l|l|l|}
\cline { 2 - 7 } \multicolumn{1}{c|}{} & Rank & $\begin{array}{l}\text { EPE } \\
\text { all }\end{array}$ & $\begin{array}{l}\text { EPE } \\
\text { matched }\end{array}$ & $\begin{array}{l}\text { EPE un- } \\
\text { matched }\end{array}$ & d0-10 & s40+ \\
\hline EpicFlow [41] & 9 & 6.285 & 3.060 & 32.56 & 5.205 & 38.02 \\
LDOF [43] & 46 & 9.116 & 5.037 & 42.34 & 6.849 & 57.30 \\
DF & 49 & 9.188 & 4.758 & 45.31 & 6.821 & 53.78 \\
DF- $\beta$ & 50 & 9.196 & 4.765 & 45.33 & 6.829 & 53.88 \\
IPOL-Brox [30] & 51 & 9.198 & 4.869 & 44.48 & 6.856 & 53.96 \\
HS [1] & 53 & 9.610 & 5.419 & 43.73 & 7.950 & 58.27 \\
DF-Auto & 54 & 9.723 & 5.200 & 46.59 & 7.483 & 57.74 \\
AnisoHuber [5] & 60 & 11.93 & 7.323 & 49.37 & 9.464 & 74.80 \\
\hline
\end{tabular}


Figure 10 shows several optical flows. We see that $D F$ Auto detects many details and contours in comparison with the other methods. For instance, in the Wall sequence, it preserves the details of the motion at the face and hair, and the flow is not so regularized at the contours. In this case, EpicFlow fails to detect the correct motion in two regions. In Perturbed Shaman 1, EpicFlow provides good results at discontinuities. $D F$-Auto also provides good results, better than $L D O F$ and IPOL-Brox. With Temple 1, it fails to detect the motion of the little dragon. This is due to the problem of small structures with large displacements, for which the pyramidal structure is not suitable.

\section{Discussion}

In this work, we studied several discontinuity-preserving techniques in optical flow methods. Our study focused on the use of decreasing functions and anisotropic diffusion tensors. We analyzed the flow instability problems that arise when decreasing functions are used in the regularization term. This has led us to propose two efficient strategies to overcome this situation.

In the experiments, we demonstrated the capabilities of these strategies and the benefits and drawbacks with respect to the Brox method. We showed their superior performance at flow discontinuities. The experiments also showed that the problem of instabilities is not anecdotal, since they usually appear in any kind of sequence. The analysis of the error evolution with respect to the discontinuity parameter demonstrated that our proposals help to correct this problem. The segmentation of the flow is better in general. The numerical comparisons showed that the increase in accuracy is not so relevant with respect to the Brox method. This is probably due to the introduction of higher errors at occlusions.

Another advantage of these discontinuity-preserving strategies is that they are very easy to implement. Our first proposal has shown to be very stable, providing very competitive solutions. Even, in many sequences, we have seen that it consistently attains the best results. Nevertheless, it is still necessary to choose the parameter.

The automatic approach computes the parameter that best adapts to the varying range of image gradients. As we have seen in the experiments, it provides solutions which are also very competitive. Without any user intervention, it usually obtains solutions which are close to the best results. Thus, we may consider that this is the most reliable strategy.

One possible improvement is to combine both strategies. In this case, we can choose a smaller $\tau$ in $f\left(\nabla I_{1}\right)$ (4), so that we may detect more discontinuities. The use of $\beta$ will still ensure a good stability.

The use of robust anisotropic diffusion tensors, like in the $R A D T$ method, also has an interesting behavior: It is very stable and it hardly introduces any instability. This is due to the fact that the regularization is always present in the isocontour direction. It can also detect some details that the other methods do not, such as in Alley 1 or Grove2. Its main drawbacks are that the motion contours are typically smoother, the accuracy is smaller than in the other strategies and it is a more complex approach.
This kind of regularization schemes fails to detect all the motion discontinuities if there exists a broad range of image and flow gradients. In future works, we will investigate more reliable ways of combining this information in order to determine the correct flow discontinuities.

\section{ACKNOWLEDGMENT}

This work has been partially supported by the Spanish Ministry of Science and Innovation (MICINN) through the project TIN2011-25488 and the grant ULPGC011-006 from the University of Las Palmas de Gran Canaria (ULPGC).

\section{REFERENCES}

[1] B. K. P. Horn and B. G. Schunck, "Determining optical flow," Artificial Intelligence, vol. 17, pp. 185-203, 1981.

[2] H. H. Nagel and W. Enkelmann, "An investigation of smoothness constraints for the estimation of displacement vector fields from image sequences," IEEE Transanctions on Pattern Analysis and Machine Intelligence, vol. 8, pp. 565-593, September 1986.

[3] L. Alvarez, J. Weickert, and J. Sánchez, "Reliable estimation of dense optical flow fields with large displacements," International Journal of Computer Vision, vol. 39, no. 1, pp. 41-56, 2000.

[4] D. Sun, J. P. Lewis, and M. J. Black, "Learning optical flow," in European Conference on Computer Vision (ECCV), 2008, pp. 83-97.

[5] M. Werlberger, W. Trobin, T. Pock, A. Wedel, D. Cremers, and H. Bischof, "Anisotropic huber-11 optical flow," in Proceedings of the British Machine Vision Conference (BMVC), London, UK, September 2009, to appear. [Online]. Available: http://gpu4vision.icg.tugraz.at/ papers/2009/werlberger_bmvc2009.pdf

[6] H. Zimmer, A. Bruhn, and J. Weickert, "Optic flow in harmony," Faculty of Mathematics and Computer Science. Universität des Saarlandes, Tech. Rep. 3, 2011. [Online]. Available: http://dx.doi.org/ 10.1007/s11263-011-0422-6

[7] M. Proesmans, L. van Gool, E. Pauwels, and A. Oosterlinck, "Determination of optical flow and its discontinuities using non-linear diffusion," in Proceedings of the third European conference on Computer Vision (Vol. II), ser. ECCV '94. Secaucus, NJ, USA: Springer-Verlag New York, Inc., 1994, pp. 295-304.

[8] M. J. Black and P. Anandan, "A framework for the robust estimation of optical flow," in Proceedings of Fourth International Conference on Computer Vision, 1993, pp. 231-236.

[9] I. Cohen, "Nonlinear Variational Method for Optical Flow Computation," in Proceedings of the 8th Scandinavian Conference on Image Analysis. Tromso, Norway: IAPR, 1993, pp. 523-530.

[10] T. Brox, A. Bruhn, N. Papenberg, and J. Weickert, "High accuracy optical flow estimation based on a theory for warping," in European Conference on Computer Vision (ECCV), ser. LNCS, T. Pajdla and J. Matas, Eds., vol. 3024. Prague, Czech Republic: Springer, May 2004, pp. 25-36.

[11] C. Zach, T. Pock, and H. Bischof, "A Duality Based Approach for Realtime TV-L1 Optical Flow," in Pattern Recognition, ser. Lecture Notes in Computer Science, F. A. Hamprecht, C. Schnörr and B. Jähne, Eds. Berlin, Heidelberg: Springer Berlin Heidelberg, 2007, vol. 4713, ch. 22, pp. 214-223. [Online]. Available: http: //dx.doi.org/10.1007/978-3-540-74936-3_22

[12] L. Álvarez, J. Esclarín, M. Lefébure, and J. Sánchez, "A pde model for computing the optical flow," in XVI Congreso de Ecuaciones Diferenciales y Aplicaciones, C.E.D.Y.A. XVI, Las Palmas de Gran Canaria, Spain, 1999, pp. 1349-1356.

[13] L. Xu, J. Jia, and Y. Matsushita, "Motion detail preserving optical flow estimation.” IEEE Trans. Pattern Anal. Mach. Intell., vol. 34, no. 9, pp. 1744-1757, 2012.

[14] J. Xiao, H. Cheng, H. Sawhney, C. Rao, and M. Isnardi, "Bilateral filtering-based optical flow estimation with occlusion detection," in Computer Vision - ECCV 2006, ser. Lecture Notes in Computer Science, A. Leonardis, H. Bischof, and A. Pinz, Eds. Springer Berlin Heidelberg, 2006, vol. 3951, pp. 211-224. [Online]. Available: http://dx.doi.org/10.1007/11744023 17

[15] M. Werlberger, T. Pock, and H. Bischof, "Motion estimation with nonlocal total variation regularization," in IEEE Conference on Computer Vision and Pattern Recognition (CVPR), San Francisco, CA, USA, June 2010, pp. 2464-2471. 
[16] R. Ranftl, K. Bredies, and T. Pock, "Non-local total generalized variation for optical flow estimation," in Computer Vision ECCV 2014, ser. Lecture Notes in Computer Science, D. Fleet, T. Pajdla, B. Schiele, and T. Tuytelaars, Eds. Springer International Publishing, 2014, vol. 8689, pp. 439-454. [Online]. Available: http://dx.doi.org/10.1007/978-3-319-10590-1_29

[17] N. Monzón, J. Sánchez, and A. Salgado, "Efficient mechanism for discontinuity preserving in optical flow methods," in Image and Signal Processing, ser. Lecture Notes in Computer Science, A. Elmoataz, O. Lezoray, F. Nouboud, and D. Mammass, Eds. Springer International Publishing, 2014, vol. 8509, pp. 425-432. [Online]. Available: http://dx.doi.org/10.1007/978-3-319-07998-1_49

[18] A. Ayvaci, M. Raptis, and S. Soatto, "Sparse occlusion detection with optical flow," International Journal of Computer Vision, vol. 97, no. 3, pp. 322-338, May 2012. [Online]. Available: http://dx.doi.org/10.1007/s11263-011-0490-7

[19] J. Sánchez, A. Salgado, and N. Monzón, "Preserving Accurate Motion Countours with Reliable Parameter Selection," in IEEE International Conference on Image Processing (ICIP2014). IEEE International Conference on Image Processing (ICIP2014), 2014, pp. 209-213.

[20] P. Perona and J. Malick, "Scale-space and edge detection using anisotropic diffusion," IEEE Transactions on Pattern Analasys and Machine Intelligence, vol. 12, pp. 629-629, July 1990.

[21] L. I. Rudin, S. Osher, and E. Fatemi, "Nonlinear total variation based noise removal algorithms," Physica D, vol. 60, pp. 259268, November 1992. [Online]. Available: http://dx.doi.org/10.1016/ 0167-2789(92)90242-F

[22] M. J. Black, G. Sapiro, D. H. Marimont, and D. Heeger, "Robust anisotropic diffusion," Trans. Img. Proc., vol. 7, no. 3, pp. 421-432, Mar. 1998.

[23] J. Weickert, "A review of nonlinear diffusion filtering," in Proceedings of the First International Conference on Scale-Space Theory in Computer Vision, ser. SCALE-SPACE '97. London, UK, UK: Springer-Verlag, 1997, pp. 3-28.

[24] —, Anisotropic diffusion in image processing. Stuttgart, Germany: Teubner-Verlag, 1998, vol. 1.

[25] J. Bigun and G. H. Granlund, "Optimal orientation detection of linear symmetry," in Proc. First Int'l Conf. Comput. Vision, 1987, pp. 433-438.

[26] C. Tomasi and R. Manduchi, "Bilateral filtering for gray and color images." in ICCV, 1998, pp. 839-846.

[27] M. J. Black and P. Anandan, "The robust estimation of multiple motions: Parametric and piecewise-smooth flow fields," Computer Vision and Image Understanding, vol. 63, no. 1, pp. 75 - 104, 1996. [Online]. Available: http://www.sciencedirect.com/science/article/ B6WCX-45N4RPR-28/2/ffc21a4d649cf9ccef82ec5d7b840e25

[28] A. Wedel, D. Cremers, T. Pock, and H. Bischof, "Structure- and motion-adaptive regularization for high accuracy optic flow," in Proceedings of IEEE International Conference on Computer Vision, Sep. 2009, pp. 1663-1668. [Online]. Available: http://dx.doi.org/10. 1109/ICCV.2009.5459375

[29] L. Xu, J. Jia, and Y. Matsushita, "Motion Detail Preserving Optical Flow Estimation," in IEEE Conference on Computer Vision and Pattern Recognition (CVPR), June 2010, pp. 1293-1300.

[30] J. Sánchez, N. Monzón, and A. Salgado, "Robust Optical Flow Estimation," Image Processing On Line, vol. 3, pp. 252-270, 2013.

[31] J. Sánchez, E. Meinhardt-Llopis, and G. Facciolo, "TV-L1 Optical Flow Estimation," Image Processing On Line, vol. 2013, pp. 137-150, 2013. http://dx.doi.org/10.5201/ipol.2013.26.

[32] H. Zimmer, A. Bruhn, J. Weickert, L. Valgaerts, A. Salgado, B. Rosenhahn, and H.-P. Seidel, "Complementary optic flow," in Proceedings of the 7th International Conference on Energy Minimization Methods in Computer Vision and Pattern Recognition, ser. EMMCVPR '09. Berlin, Heidelberg: Springer-Verlag, 2009, pp. 207-220.

[33] K.-J. Yoon and I.-S. Kweon, "Adaptive support-weight approach for correspondence search." IEEE Transactions on Pattern Analysis and Machine Intelligence, vol. 28, no. 4, pp. 650-656, 2006.

[34] D. Sun, S. Roth, and M. J. Black, "A quantitative analysis of current practices in optical flow estimation and the principles behind them," International Journal of Computer Vision, vol. 106, no. 2, pp. 115-137, 2014.

[35] E. Mémin and P. Pérez, "Dense estimation and object-based segmentation of the optical-flow with robust techniques," IEEE Transactions on Image Processing, vol. 7, no. 5, pp. 703-719, May 1998.

[36] D. Sun, E. Sudderth, and M. J. Black, "Layered segmentation and optical flow estimation over time," in IEEE Conf. on Computer Vision and Pattern Recognition (CVPR). IEEE, 2012, pp. 1768-1775.
[37] M. Unger, M. Werlberger, T. Pock, and H. Bischof, "Joint motion estimation and segmentation of complex scenes with label costs and occlusion modeling." in CVPR. IEEE, 2012, pp. 1878-1885.

[38] J. Sánchez, A. Salgado, and N. Monzón, "Optical flow estimation with consistent spatio-temporal coherence models," in International Conference on Computer Vision Theory and Applications (VISAPP). Institute for Systems and Technologies of Information, Control and Communication, 2013, pp. 366-370.

[39] D. J. Butler, J. Wulff, G. B. Stanley, and M. J. Black, "A naturalistic open source movie for optical flow evaluation," in European Conf. on Computer Vision (ECCV), ser. Part IV, LNCS 7577, A. Fitzgibbon et al. (Eds.), Ed. Springer-Verlag, Oct. 2012, pp. 611-625.

[40] S. Baker, D. Scharstein, J. P. Lewis, S. Roth, M. J. Black, and R. Szeliski, "A database and evaluation methodology for optical flow," in International Conference on Computer Vision, 2007, pp. 1-8.

[41] J. Revaud, P. Weinzaepfel, Z. Harchaoui, and C. Schmid, "Epicflow: Edge-preserving interpolation of correspondences for optical flow," arXiv preprint arXiv:1501.02565, 2015.

[42] A. Wedel, T. Pock, C. Zach, H. Bischof, and D. Cremers, "An improved algorithm for tv-11 optical flow," in Statistical and Geometrical Approaches to Visual Motion Analysis. Springer Berlin Heidelberg, 2009 , pp. 23-45.

[43] T. Brox and J. Malik, "Large displacement optical flow: Descriptor matching in variational motion estimation," IEEE PAMI, vol. 33, no. 3, pp. 500-513, 2011. 

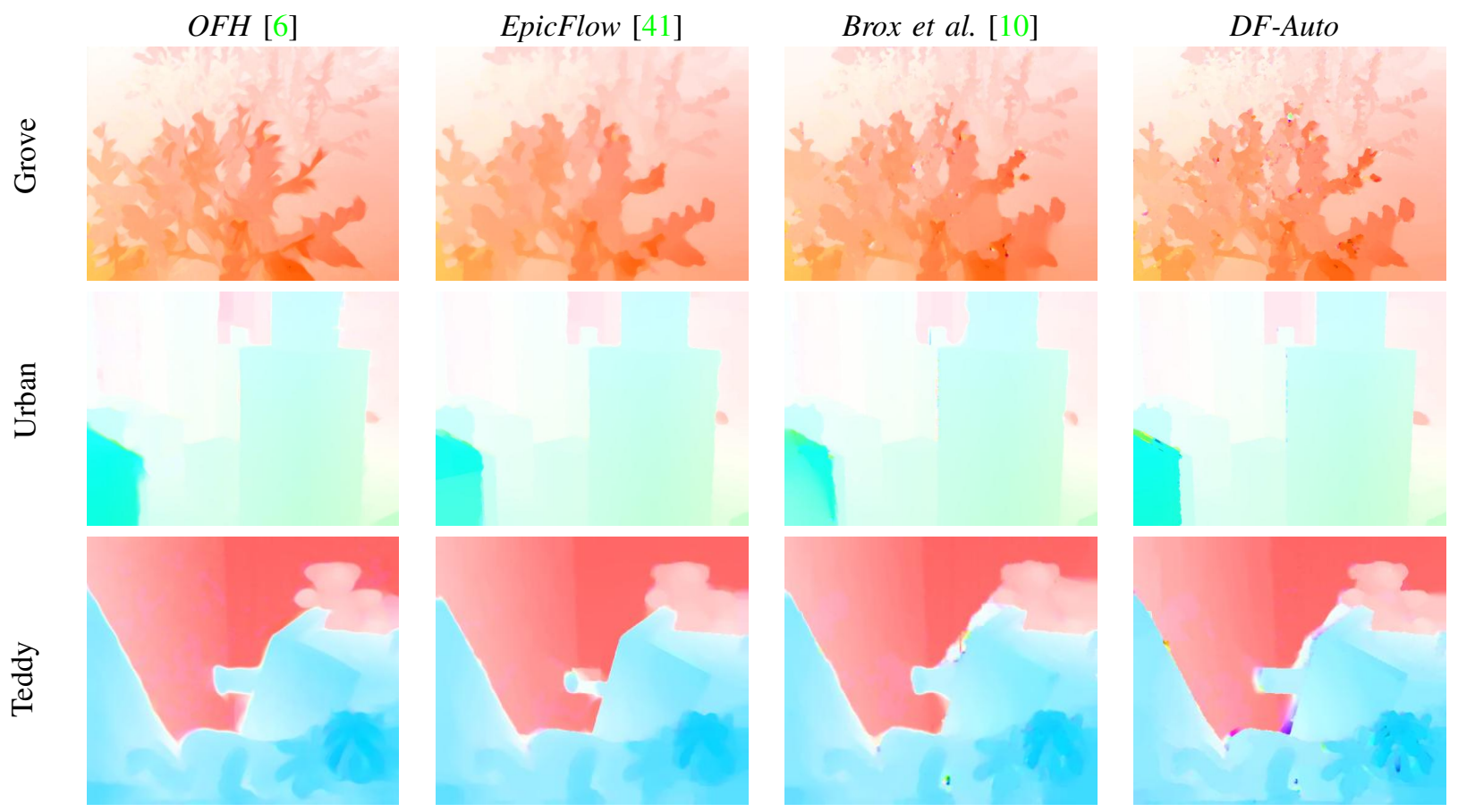

Fig. 9. Comparison of flow fields obtained with OFH, EpicFlow, IPOL-Brox and DF-Auto methods using the Middlebury evaluation dataset.

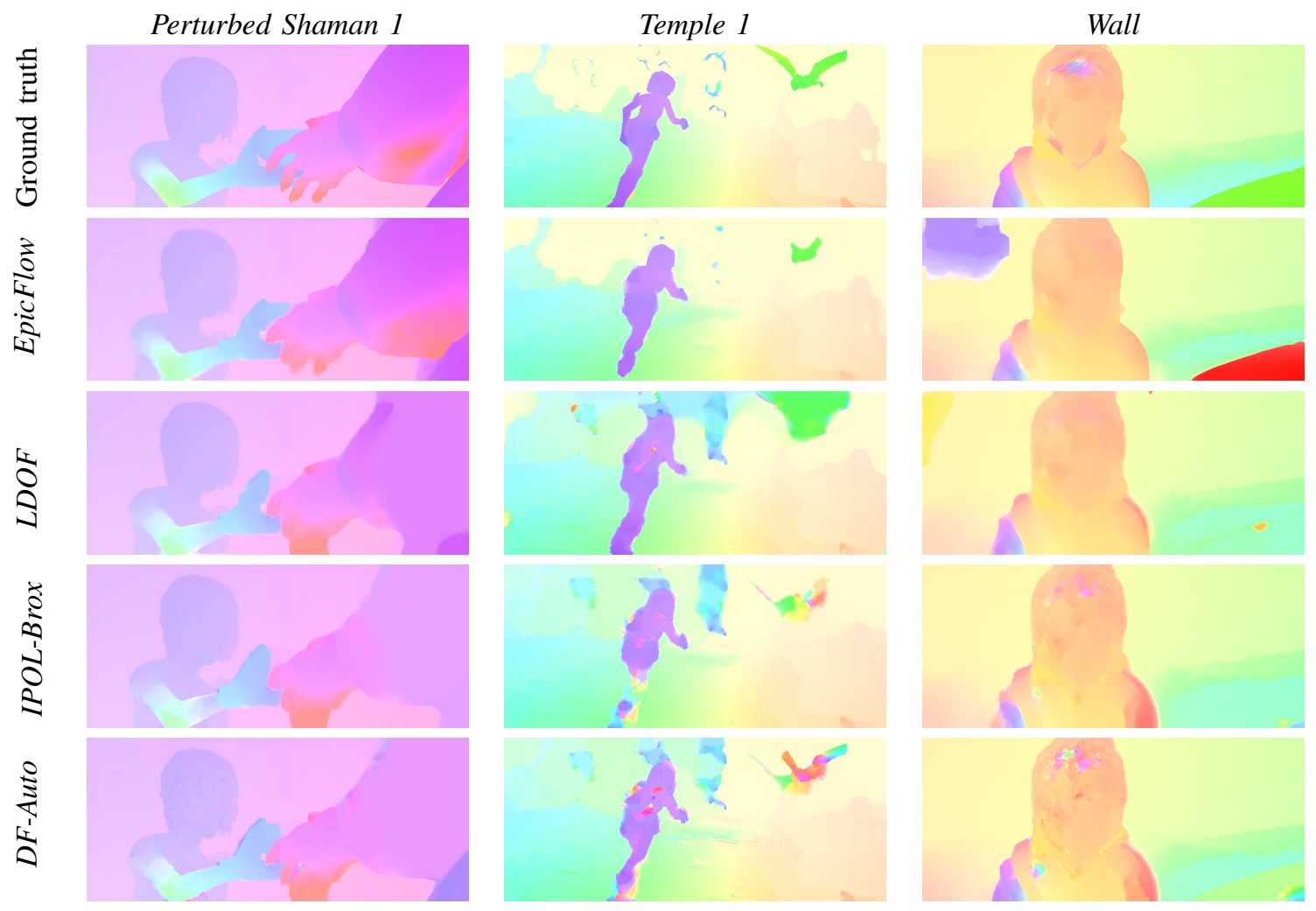

Fig. 10. Comparison of flow fields obtained with the Ground Truth, EpicFlow, LDOF, IPOL-Brox and DF-Auto methods using the Sintel Clean test dataset. 